\title{
¿Planifican los tutores la formación de sus residentes? Investigación realizada en la especialidad de Medicina Familiar y Comunitaria
}

\author{
Eduardo Romero-Sánchez, Iñigo Anza-Aguirrezabala, José Saura-Llamas
}

Introducción. ¿Planifican los tutores la formación de sus residentes? Si es así, ¿cómo lo hacen?, ¿existen diferencias entre lo explicitado en los programas formativos de las distintas especialidades y lo que ocurre realmente en la práctica diaria?, ¿están formados los tutores para planificar su docencia desde el punto de vista pedagógico?

Sujetos y métodos. Para dar respuesta a estos interrogantes se ha llevado a cabo un estudio exploratorio de corte descriptivo. En él se ha diseñado, validado y presentado un cuestionario que recoge la opinión contrastada tanto de tutores como de sus respectivos residentes. La población la componen el total de los médicos residentes R1 (promoción 2006-2010; $n=57$ ) y R3 (promoción 2004-2007; $n=58$ ) de Medicina Familiar y Comunitaria de la Región de Murcia, y el total de la población de sus respectivos tutores: tutores de R1 $(n=57)$ y tutores de R3 $(n=58)$. Han participado 26 centros de salud que gestionan las tres unidades docentes de la región. Los datos se han analizado mediante el programa SPSS v. 14.0.

Resultados y conclusiones. Aunque en la actividad docente de los tutores está muy presente la espontaneidad y la improvisación, existen importantes elementos de programación que hay que saber extraer e identificar porque son propios de un contexto práctico de formación. Se reconoce una mayor satisfacción de los implicados cuando el proceso formativo está más planificado.

Palabras clave. Educación médica. Formación de posgrado. Medicina familiar y comunitaria. Plan individual de formación. Residente. Tutor.

Do tutors plan the formation of their residents? A study realized in the specialty of Familiar and Community Medicine

Introduction. Do tutors plan the formation of their residents?; and if so, how do they do it?, are there any differences between the content of the training programs of the different specialties and what actually happens in daily practice?, are they trained to plain their teaching from a pedagogical point of view?

Subjects and methods. To answer these questions we have developed an exploratory study-descriptive. It has been designed, validated and passed a questionnaire that reflects the views contrasted of tutors and their respective residents. The population is composed by the total of the residents R1 (promotion 2006-2010; $n=57$ ) and R3 (promotion 20042007; $n=58$ ) of Familiar and Community Medicine of the Region of Murcia and the total of the population of their respective tutors: tutors of R1 $(n=57)$ and tutors of R3 $(n=58)$. In this study there are involved 26 health centers of the three teaching units in the region. The exploitation of the information has been developed through statistical analysis with SPSS software v. 14.0.

Results and conclusions. Though in the educational activity of the tutors spontaneity and improvisation are very present, there are important elements of programming which require some knowledge to be extracted and identified because they belong to a practical context of formation. A major satisfaction of the ones involved is recognized when the formative process is more planned.

Key words. Family and community medicine. Individual training plan. Medical education. Postgraduate training. Resident. Tutor.

\section{Introducción}

Este trabajo es parte de un estudio más amplio [1] que describe y analiza las prácticas en el diseño y la puesta en marcha de los procesos formativos que se establecen entre tutor y residente en el contexto profesional que constituye el sistema de médicos internos residentes (MIR). En dicha investigación se
Departamento de Teoría e Historia de la Educación; Facultad de Educación; Universidad de Murcia (E. Romero-Sánchez). Unidad Docente de Medicina Familiar y Comunitaria; Gerencia del Área III de Salud de Lorca (I. AnzaAguirrezabala). Unidad Docente de Medicina Familiar y Comunitaria; Gerencia de Atención Primaria de Murcia (J. Saura-Llamas). Murcia, España.

Correspondencia:

Prof. Eduardo Romero Sánchez. Departamento de Teoría e Historia de la Educación. Facultad de Educación. Universidad de Murcia. Campus de Espinardo. E-30100 Murcia.

E-mail:

eromero@um.es

Conflicto de intereses: No declarado.

Conflict of interests: None declared.

(c) 2012 Educación Médica 
profundiza en el entramado que conforma la planificación de la formación médica especializada: normativa reguladora, concepto de programación didáctica o plan de formación, funciones y utilidades, principales elementos y niveles de concreción curricular y experiencias más importantes desarrolladas hasta el momento en lo que a la planificación del médico residente se refiere.

Quizás la mayor aportación o fortaleza del trabajo resida en la mirada pedagógica que vierte sobre los procesos implicados en el diseño y desarrollo de la programación formativa del residente, en este caso, de medicina de familia. Los resultados han permitido obtener información relevante sobre la práctica real de los tutores en materia de planificación, y las conclusiones extraídas permitirán ofrecer orientaciones prácticas que ayuden a mejorar la planificación como una función docente de tutores y responsables docentes.

Para la fundamentación y contextualización del estudio se ha realizado una exhaustiva revisión en educación médica, utilizándose los principales referentes teóricos y bibliográficos en la materia [2-11].

\section{Justificación teórica y normativa}

En la formación médica especializada, como en cualquier otra actividad organizada que pretenda unos objetivos, es preciso planificar. Lo contrario deja tanto al tutor como al residente a la deriva en el inmenso océano de la improvisación, que quizás sea más apropiada para otras facetas creativas y lúdicas del ser humano, pero no tanto para las que exigen organización y sistematización, como es el caso de los procesos de enseñanza-aprendizaje. Por tanto, el propósito esencial de la planificación es el de constituir una herramienta útil, un aporte significativo a la hora de proyectar cómo y qué habrá de enseñarse, una organización optimizadora y facilitadora del trabajo que se realizará durante el período formativo.

Tanto en la Orden de 22 de junio de 1995, por la que se regulan las Comisiones de Docencia [12], como en la Ley de Ordenación de las Profesiones Sanitarias (LOPS) y en el Real Decreto 183/2008 [13] aparecen referencias explícitas al tema de la planificación de la formación de los residentes. Principalmente, cuando se hace alusión a las funciones de las Comisiones de Docencia y del tutor, se indica que la actividad profesional de los residentes debe planificarse de modo que se integre en la vida normal de los centros. Por tanto, según la normativa vigente, corresponde al tutor la propuesta a la Comisión de Docencia de los planes individuales de formación para cada uno de los especialistas en formación a su cargo.

Para poder desempeñar esta función planificadora, el tutor, aunque cuenta con un instrumento general como es el programa oficial de su especialidad, deberá realizar una adaptación de éste para adecuarlo a las características de su centro y de los residentes a su cargo. En otras palabras, los tutores, a la hora de planificar, deben pensar cuidadosamente cuáles son los conceptos básicos involucrados en su trabajo diario, y para ello deben prestar atención a dos puntos de vista: el que marca el programa de la especialidad y el que podría planificarse para resolver las necesidades de aprendizaje de cada residente individual, de forma personalizada. Así, la relación tutor-residente permite al primero un desarrollo individualizado del programa formativo, de modo que el especialista en formación pueda crecer a partir de sus puntos fuertes y corregir sus debilidades.

Pero el diseño y desarrollo de un plan de formación es una tarea de considerable trabajo y nada sencilla, que precisa un mínimo apoyo y estructura docentes y, sobre todo, la voluntad y preparación de los tutores en el desempeño de una serie de competencias para las cuales no sabemos si se encuentran preparados. Por ello no es de extrañar que la mayoría de los tutores prefieran centrar la energía del aprendizaje sobre los problemas que surgen en el día a día, más que en definir su propio plan de formación o incluso poner en práctica el propuesto en el ámbito estatal.

Sin embargo, ya hay algo de camino recorrido. Se han desarrollado en nuestro país varias experiencias docentes en los últimos años en el seno de unidades docentes y diferentes instituciones. Aunque varía la estrategia de intervención pedagógica o el instrumento que se usa en cada caso (negociación del currículo, contrato de formación, portafolio docente, tutorización activa continua...), mantiene prácticamente el mismo esquema: la definición de la competencia final y de los objetivos, la asignación de las tareas, y la evaluación y opinión de los residentes.

\section{Objetivo}

Tras la descripción del contexto en el que surge y se inscribe esta investigación, se justifica y delimita el problema de estudio a una especialidad concreta -Medicina Familiar y Comunitaria (MFyC)-, a un contexto específico -la Región de Murcia- y a un momento formativo preciso - la etapa inicial tanto del primer como del tercer año del período 
de estancia formativa del residente en el centro de salud-.

Se plantean una serie de interrogantes de carácter general a los cuales el trabajo pretende dar respuesta: ¿planifican los tutores la formación de sus residentes?; si es así, ¿cómo lo hacen?, ¿existen diferencias entre lo explicitado en el programa formativo de la especialidad y lo que ocurre realmente en la práctica diaria?, ¿están formados los tutores para planificar su docencia desde el punto de vista pedagógico?

Más específicamente, el estudio pretende describir cómo se diseña y se desarrolla el plan individual de formación del residente de MFyC en los centros de salud docentes de la Región de Murcia.

\section{Sujetos y métodos}

En este apartado se expone todo el proceso metodológico seguido en el trabajo de campo: el diseño metodológico, las variables analizadas, el proceso de construcción y validación del instrumento utilizado, la población participante, el proceso de recogida de los datos y su tratamiento.

\section{Diseño metodológico}

La presente investigación se enmarca dentro de los estudios de corte descriptivo que, como señalan algunos de los autores consultados [14,15], son útiles en el conocimiento y exploración de determinados ámbitos sociales y, en particular, en la investigación educativa.

\section{Instrumento}

Se ha empleado un cuestionario anónimo autoadministrado con cuatro versiones: una dirigida a residentes de primer año (R1), otra a residentes de tercer año (R3), otra a los tutores de los R1 y una cuarta a los tutores de los R3. Se establecen unos conjuntos o grupos de variables que van a guiar la construcción del cuestionario:

- Primer grupo. Se relaciona con información de carácter demográfico y académico sobre los participantes que responden al cuestionario. Se trataría, por tanto, de variables de identificación personal, como sexo, edad, elección de MFyC como primera opción, unidad docente de pertenencia...

- Segundo grupo. Integra todas aquellas variables que hacen alusión a los elementos o aspectos que definen el plan individual de formación del residente: objetivos formativos, ritmos de aprendi- zaje, reuniones de seguimiento, criterios de evaluación...

- Tercer grupo. Se refiere a aspectos de valoración de la satisfacción del proceso formativo y de implicación en él. Se trata de una valoración final que recoge las percepciones sobre el programa, el tutor, el aprendizaje realizado, los recursos...

\section{Validación}

El cuestionario, desde la versión inicial hasta la final, se ha sometido a una serie de procesos de validación a partir de los cuales se han introducido cambios antes de considerarlo definitivo. Primeramente se ha hecho una validación cualitativa de contenido por parte de jueces o expertos. Con posterioridad, se ha realizado un ensayo piloto (de las cuatro versiones del cuestionario) en el Área III, que corresponde a la zona de salud de Lorca. Una vez asegurada la validez de contenido y de construcción del cuestionario era el momento de comprobar la fiabilidad del instrumento. Para ello se calculó el valor $\alpha$ de Cronbach, obteniéndose una fiabilidad muy elevada $(0,88)$.

\section{Población}

La población la componen 57 médicos R1 (promoción 2006-2010) y 58 médicos R3 (promoción 20042007) de MFyC de la Región de Murcia, junto con el total de la población de sus respectivos tutores: 57 tutores de R1 (T1) y 58 tutores de R3 (T3). Se trabaja con todo el colectivo sin necesidad de seleccionar una muestra al no ser excesivamente grande $\mathrm{y}$ encontrarse a nuestro alcance.

El hecho de haber escogido, dentro de la especialidad, a los médicos residentes de primer y tercer año, y sus respectivos tutores, se explica por dos aspectos: por un lado, porque están en las fases inicial y final de su proceso de residencia, y por otro, porque constituyen sus principales estancias formativas en el centro de salud, momentos clave para el diseño del plan de formación objetivo de este trabajo.

\section{Emplazamiento}

Han participado todos los centros de salud docentes que contaban, en el momento de realización del estudio, con las poblaciones de tutores y residentes requeridas: 26 centros, distribuidos en su demarcación territorial por las distintas áreas de salud de la comunidad autónoma y gestionados por las cinco gerencias de atención primaria y las tres unidades docentes de la región (Murcia, Cartagena y Lorca). 


\section{Tratamiento de los datos}

Para el tratamiento de los datos obtenidos en el cuestionario nos hemos servido del análisis estadístico, que es el método más apropiado para presentar la información cuantitativa de manera comprensiva. La explotación de los datos se ha realizado con el programa SPSS v.14.0 para obtener el análisis de frecuencia en porcentajes y el cruce de los datos.

\section{Resultados}

En general, tutores y residentes reconocen la práctica de una evaluación diagnóstica efectuada por parte del tutor a su residente tanto en los primeros días de su estancia inicial como R1 en el centro de salud, como también al inicio de su rotación como R3. Ambos colectivos destacan, como elementos más importantes, el conocer la experiencia previa y las expectativas formativas del residente.

La mayor parte de los participantes de esta investigación reconocen que el tutor comunicó al residente lo que debía aprender durante la rotación, una indicación de los objetivos docentes que casi siempre se produjo de forma verbal y que fue bien valorada tanto en términos de cantidad como de calidad por ambos colectivos.

En el diseño del plan de formación del residente, tanto tutores como R1 y R3 otorgan a las necesidades del residente y al criterio y experiencia del tutor un mayor peso que al resto de elementos explorados (expectativas formativas del residente y programa de la especialidad). Coinciden los T1 y los T3 en conferir mayor importancia al programa de la especialidad que sus respectivos residentes.

En general, tanto tutores como residentes manifiestan que en el proceso formativo del residente se tuvieron en cuenta sus ritmos de aprendizaje de una manera bastante frecuente.

Todos los participantes del estudio coinciden en destacar el aprovechamiento de las experiencias cotidianas del día a día como el recurso formativo más utilizado por los tutores, por encima de los casos clínicos y de las actividades específicamente diseñadas con fines formativos.

En general, existe plena coincidencia entre tutores y residentes en destacar el aprendizaje en el contexto real o de campo y el autoaprendizaje como los métodos más empleados por el residente durante su estancia inicial en el centro de salud de entre los propuestos por el programa oficial de la especialidad. Se advierte también que los R1 y R3 son más conscientes de su autoaprendizaje que sus tutores.
Tanto T1 como T3 aseguran haber mantenido más reuniones de seguimiento o de valoración formativa con sus residentes que las que reconocen haber tenido estos últimos. La mayor parte de las reuniones ha sido de tipo informal, siendo el tema más abordado en ellas el referido a las dificultades o problemas surgidos al residente en su formación hasta ese momento. En la mayor parte de los casos no suelen planificarse estas reuniones mediante un calendario o cronograma, y se realizan más bien sobre la marcha.

Existe tanto entre residentes como entre tutores un porcentaje bastante elevado de coincidencia que reconoce no haber mantenido sesiones formativas de feedback tutor-residente. Es preciso trabajar en este sentido porque este tipo de encuentros de valoración del progreso seguido por el residente constituye el mejor modo para detectar si los objetivos se están alcanzando de forma adecuada y, en caso contrario, realizar los reajustes necesarios para que mejore su proceso formativo.

Se debe perfilar mejor la comunicación y clarificación de los criterios de evaluación por parte del tutor al residente, pues existen porcentajes muy elevados que reconocen no haber realizado esta tarea. Constituye una labor fundamental para que los residentes formen parte y participen en su propio proceso de evaluación, discutiendo los criterios, su aplicación y los resultados.

Tanto residentes como tutores tienden a considerar su propia participación en la toma de decisiones sobre cuestiones formativas por encima de lo considerado por su pareja docente, pero ambos compensan esta diferencia refiriendo que comparten este protagonismo por encima de su propia opinión.

En general, las enfermeras del cupo docente son las que más colaboran en la formación del residente después del propio tutor y por encima de otros profesionales del centro, como otros tutores, administrativos, trabajadores sociales, matronas, fisioterapeutas, pediatras, otros residentes, etc.

La valoración general del grado de satisfacción de tutores y de residentes es muy positiva, siendo un poco más elevada en el caso de estos últimos. En cuanto a aspectos más específicos, todos los participantes muestran una mayor satisfacción en aquellos elementos que dependen en mayor medida de la disposición y actitud del tutor: la relación mantenida, el apoyo ofrecido y el asesoramiento prestado al residente (en este orden). Por contra, son los aspectos de tipo organizativo y metodológico los que obtienen una peor valoración.

Todos los participantes manifiestan un alto grado de implicación en el proceso formativo, siendo 
un poco más elevado el manifestado por los residentes que por los tutores.

En general, tanto para tutores como para residentes, el estilo de planificación docente del tutor se caracteriza por ser algo más oportunista que planificado, pero siendo las diferencias muy poco significativas como para poder definir un perfil claro. Por otro lado, los R1 y los R3 perciben la planificación del tutor como más sistematizada y estructurada que los tutores, y éstos, a su vez, la perciben como más oportunista y espontánea que los residentes.

La carencia de recursos para la correcta planificación de la actividad docente (tiempo, espacios, formación y materiales) es mucho más percibida por los tutores que por los residentes. Quizás debería averiguarse si esta queja está fundamentada en todos los casos o es utilizada por los tutores para justificar su falta de competencia clínica y docente.

Se reconoce una mayor satisfacción de los implicados cuando el proceso formativo está más planificado, apareciendo los aspectos 'humanos' de la tutorización (asesoramiento, apoyo, relación) como más valorados en términos de satisfacción que aquellos más propios de la programación (métodos, contenidos, organización).

En términos generales, se reconoce un sistema consistente (elevada coincidencia entre tutores y residentes), rico en programación de la actividad docente y con gran variabilidad.

Según la opinión de los expertos consultados -información obtenida de las aportaciones realizadas por una serie de expertos participantes en el panel desarrollado en las primeras fases de esta investigación-, la forma en que los tutores programan su labor docente se caracteriza por la improvisación, la voluntad individual (tanto de residentes como de tutores) y la observación directa. Expresado en sus mismas palabras:

- 'Creo que el modelo teórico de programación didáctica no es explícito o suficientemente explícito en el día a día entre residente y tutor'.

- 'No creo que en la realidad ningún tutor, centro o unidad, de forma deliberada, consciente y real, se dirijan hacia las competencias que tiene que aprender el médico de familia, sino más bien a la adaptación del residente al modelo de práctica que realiza el tutor en ese centro'.

- 'Mira como hago, pregunta y aprende. Haz lo que puedas y, cuando tengas dudas, pregunta. Considero que los tutores no se plantean un modelo práctico ni teórico de programación didáctica.'

- 'Al no poseer la formación pedagógica necesaria, en la mayoría de los casos, la formación del resi- dente de MFyC se basa en actividades que no presuponen demasiada estructuración.

\section{Conclusiones}

Si bien en la actividad docente de los tutores está muy presente la espontaneidad y la improvisación, existen importantes elementos de programación que hay que saber extraer e identificar porque son propios de un contexto práctico de formación.

Una planificación de la formación individual puede aportar numerosas ventajas sobre la formación general, que básicamente se resumen en más motivación y compromiso, más conocimiento y control sobre los resultados de aprendizaje y, en definitiva, mayor calidad de la formación [16].

La incorporación del residente en la actividad del centro sin un plan de formación individual deja el aprendizaje del residente expuesto a las circunstancias azarosas del contexto, lo que se ha denominado 'aprendizaje oportunista', de modo que la presión asistencial, sus preferencias o temores, pueden hacer que finalice el período de residencia sin que su programa se haya completado en determinadas áreas o que no haya desarrollado alguna de las competencias básicas.

Las estrategias docentes basadas en una mera transmisión de la información sobre la marcha, sin haberlas planificado ni preparado, no son siempre las más idóneas. Es decir, el modelo por el cual el residente aprende por el mero hecho de acompañar a su tutor y seguir las actividades diarias para, al cabo de cuatro años de residencia, considerarse ya capacitado, no es el más conveniente.

El denominador común de lo que se presenta es una satisfacción importante del colectivo, que valora de manera muy especial el estrecho vínculo del residente con su tutor, cargado de relación interpersonal-profesional, con indudables repercusiones en un mutuo enriquecimiento. Sin duda, en los próximos años, el mantenimiento de la calidad del colectivo implicará la exigencia de un adecuado entrenamiento en metodología docente, herramientas de tutorización e incluso elementos pedagógicos aplicados a la tutorización.

Aunque es cierto que las oportunidades de aprendizaje que se producen durante la residencia dependen mucho de las características personales y profesionales del tutor y de factores como la motivación y la implicación que tengan el especialista en formación, el propio tutor y la organización en que se realiza la formación, nuestra contribución con este trabajo responde a la necesidad de homo- 
geneizar más la formación de los resientes y aumentar la competencia docente de los tutores en materia de planificación [17].

Este trabajo puede servir para ordenar mejor la función docente, pero desde sus fortalezas. Es decir, aunque es necesario adaptar y mejorar la estructura y el proceso docente, no se ha de olvidar que el sistema MIR es un sistema natural de aprendizaje cuyo éxito está más que demostrado.

Bibliografía

1. Romero E. Análisis pedagógico del plan de formación individual del residente de Medicina Familiar y Comunitaria [tesis doctoral]. Murcia: Universidad de Murcia; 2008.

2. Harden RM, Sowden S, Dunn WR. Some educational strategies in curriculum development: the SPICES model. Med Educ 1984; 18: 284-97.

3. Harden RM. Ten questions to ask when planning a course or curriculum. Med Educ 1986; 20: 356-65.

4. Harden RM. Curriculum mapping: a tool for transparent and authentic teaching and learning. AMEE Guide n. ${ }^{\circ} 21 ; 2001$.

5. Kern DE, Thomas PA, Howard DM, Bass EB. Curriculum development for medical education: a six-step approach. Baltimore: Johns Hopkins Press; 1998.

6. World Federation for Medical Education. International standards in medical education: assessment and accreditation of medical schools' educational programmes. Med Educ 1998; 32: 549-58.
7. Harden RM, Crosby JR. The good teacher is more than a lecturer: the twelve roles of the teacher. AMEE Medical Education Guide n. ${ }^{\circ} 20$. Med Teach 2000; 22: 334-47.

8. Snyder S. A program to teach curriculum development to junior faculty. Fam Med 2001; 33: 382-7.

9. Wojtczak A. Glosario de términos de educación médica. Educ Med 2003; 6 (Supl 2): 28.

10. Palés J. Planificar un currículum o un programa formativo. Educ Med 2006; 9: 59-65.

11. Vicent-García MD. Diseño del programa de formación individual: del programa oficial de la especialidad al desarrollo curricular del residente. In Cabero L, coord. Manual para tutores de MIR. Madrid: Editorial Médica Panamericana; 2007. p. 73-91.

12. Orden de 22 de junio de 1995, por la que se regulan las comisiones de docencia y los sistemas de evaluación de la formación de médicos y de farmacéuticos especialistas. BOE, n. ${ }^{\circ}$ 155, de 30 de junio de 1995.

13. Real Decreto $183 / 2008$, de 8 de febrero, por el que se determinan y clasifican las especialidades en Ciencias de la Salud y se desarrollan determinados aspectos del Sistema de Formación Sanitaria Especializada. Ministerio de Sanidad y Consumo. BOE, n. ${ }^{\circ} 45$, de 21 de febrero de 2008.

14. Bisquerra R. Metodología de la investigación educativa. Madrid: La Muralla; 2004.

15. Gall M, Gall J, Borg W. Educational research. An introduction. Boston: Pearson; 2006.

16. Ricarte JI, Martínez-Carretero JM. Métodos de enseñanza y aprendizaje en el residente. In Cabero L, coord. Manual para tutores de MIR. Madrid: Editorial Médica Panamericana; 2007.

17. Socas M. El punto de vista de los residentes en Europa. Educ Med 2005; 3: 120-7. 